\title{
The Need for Continuing Chemotherapy for Leukemic Cell Lysis Pneumopathy in Patients with Acute Myelomonocytic/Monocytic Leukemia
}

\author{
Aiko Kato, Yuichiro Ono, Yosuke Nagahata, Nobuhiko Yamauchi, Sumie Tabata, \\ Noboru Yonetani, Akiko Matsushita and Takayuki Ishikawa
}

\begin{abstract}
Although fatal pulmonary complications frequently occur during the course of acute leukemia, a minor proportion of the complications are due to leukemia itself. Infections, drug reactions and concomitant medical conditions are the major causes of respiratory distress in leukemic patients. We treated four patients with acute myeloid leukemia complicated by leukemic cell lysis pneumopathy (LCLP). All of the patients had leukemia of monocytoid origin and their respiratory function deteriorated soon after chemotherapy initiation. Although two of the patients required mechanical ventilation, all four improved after continued chemotherapy. Our experience indicates that, in cases of LCLP, chemotherapy should be continued with maximal respiratory support.
\end{abstract}

Key words: leukemic cell lysis pneumopathy, acute myelomonocytic/monocytic leukemia, respiratory support, corticosteroids

(Intern Med 52: 1217-1221, 2013)

(DOI: 10.2169/internalmedicine.52.9117)

\section{Introduction}

Patients with acute leukemia frequently experience early respiratory events complicated by respiratory failure (1). These complications can be caused by a variety of conditions, including infection, pulmonary edema, alveolar hemorrhage, drug reactions and leukemic involvement (2). Pulmonary leukemic cell infiltration is quite common, while symptomatic pulmonary disease caused by leukemia is not (3). Leukemic cell lysis pneumopathy (LCLP) is an uncommon mechanism of respiratory distress associated with leukemia that can be fatal (4-6). Azoulay et al. reported 20 patients with respiratory failure related to leukemic pulmonary involvement due to leukostasis or leukemic infiltration that showed remarkable features: it was exclusive to monocyte-derived leukemia and was associated with rapidly progressive respiratory distress and a deteriorating respiratory status just after chemotherapy initiation (7). Ten of the 20 patients died, despite receiving aggressive respiratory support (including mechanical ventilation). Azoulay and colleagues also reported that the clinical manifestations of all of the patients were consistent with LCLP. Unfortunately, there are limited reports regarding the management of LCLP, although early administration of dexamethasone has been recommended (8).

We encountered four patients with acute monocytic or myelomonocytic leukemia complicated by LCLP. We herein present relevant clinical information and discuss the strategy for the management of LCLP.

\section{Case Reports}

Table shows the clinical features of the patients. All of the patients were diagnosed with acute monocytic or myelomonocytic leukemia and their respiratory functions deteriorated within 24 hours of chemotherapy initiation.

\section{Patient No. 1}

A 77-year-old woman was referred to us after a routine 
Table. The Clinical Features of the Patients

\begin{tabular}{|c|c|c|c|c|c|}
\hline Patient No. & & 1 & 2 & 3 & 4 \\
\hline Age(years), gender & & 73 , female & 48 , male & 66 , male & 15 , female \\
\hline FAB classification & & M4 & M5 & M4E & M4E \\
\hline Karyotype & & $46, \mathrm{XX}$ & $46, X Y$ & $\begin{array}{c}46, X Y \\
\operatorname{inv}(16)(\mathrm{p} 13.1 \mathrm{q} 22)\end{array}$ & $\begin{array}{c}46, X X \\
\operatorname{inv}(16)(p 13.1 q 22)\end{array}$ \\
\hline Body temperature at admission $\left({ }^{\circ} \mathrm{C}\right)$ & & 36.5 & 39.5 & 37.3 & 38.4 \\
\hline Dyspnea at admission & & - & - & + & + \\
\hline \multicolumn{6}{|l|}{ Laboratory data } \\
\hline & WBC $(/ \mu \mathrm{L})$ & 5,500 & 138,400 & 107,400 & 7,900 \\
\hline & PB blast (\%) & 11 & 85 & 52 & 59 \\
\hline & PB monocyte $(\%)$ & 44 & 3 & 32 & 5 \\
\hline & $\mathrm{RBC}\left(\times 10^{4} / \mu \mathrm{L}\right)$ & 310 & 405 & 195 & 311 \\
\hline & $\mathrm{Hb}(\mathrm{g} / \mathrm{dL})$ & 9.1 & 12.3 & 7.1 & 9.8 \\
\hline & Plt $\left(\times 10^{4} / \mu \mathrm{L}\right)$ & 3.2 & 33.3 & 2.4 & 2.9 \\
\hline & $\mathrm{LDH}(\mathrm{IU} / \mathrm{mL})$ & 284 & 1,226 & 937 & 310 \\
\hline & $\mathrm{CRP}(\mathrm{mg} / \mathrm{dL})$ & 2.3 & 2.3 & 3 & 3.6 \\
\hline Chemotherapy & & AraC+DNR, MEC & AraC+IDR & $\mathrm{AraC}+\mathrm{DNR}+\mathrm{VP}-16$ & VP-16+AraC+Mit \\
\hline Mechanical ventilation & & NIV & - & intubation & - \\
\hline Time from chemotherapy initiation & & 18 hours/5 hours & 8 hours & 4 hours & 2 hours \\
\hline Steroid for respiratory distress & & none & $\begin{array}{c}\text { mPSL } 80 \mathrm{mg} \\
\text { day } 1-9\end{array}$ & $\begin{array}{c}\text { mPSL } 125 \mathrm{mg} \\
\text { day } 8-12\end{array}$ & $\begin{array}{l}\text { mPSL } 80 \mathrm{mg} \\
\text { day } 1-3\end{array}$ \\
\hline Duration of $\mathrm{O}_{2}$ supply & & 9 days $/ 14$ days & 15 days & 12 days & 7 days \\
\hline Outcome & & alive: $\mathrm{CR}$ & alive: $\mathrm{CR}$ & alive: $\mathrm{CR}$ & alive: $\mathrm{CR}$ \\
\hline Follow up (months) & & 23 & 25 & 15 & 9 \\
\hline
\end{tabular}

FAB: French-American-British hematology group, PB: peripheral blood, AraC: cytarabine, IDR: idarubicin, DNR: doxorubicin, Mit: mitxantrone, NIV: non-invasive ventilation, mPSL: methylprednisolone, CR: complete remission

laboratory examination ordered by her doctor revealed mild anemia and thrombocytopenia. Upon hospital admission, her white blood cell count was $5,500 \mu \mathrm{L}$ with $11 \%$ myeloblasts and $44 \%$ monocytes. Following bone marrow aspiration, she was diagnosed with acute myelomonocytic leukemia and treated with cytarabine and daunorubicin (cytarabine, 100 $\mathrm{mg} / \mathrm{m}^{2}$, administered on Days 1-5; daunorubicin, $30 \mathrm{mg} / \mathrm{m}^{2}$, administered on Days 1-3). On the second day of treatment, she became progressively dyspneic and required noninvasive mechanical ventilation (Fig. 1-1). Chemotherapy was continued, and her respiratory status improved in parallel with a reduction in blast cell numbers. Unfortunately, the first induction chemotherapy failed, and the patient's respiratory function again deteriorated as the monoblastic cell count increased. She received MEC therapy (mitoxantrone, $6 \mathrm{mg} / \mathrm{m}^{2}$, administered on Days 1-2; etoposide, $100 \mathrm{mg} / \mathrm{m}^{2}$, administered on Days 1-5; and cytarabine, $1 \mathrm{~g} / \mathrm{m}^{2}$, administered on Days 1-5); however, she became rapidly dyspneic five hours after treatment initiation and again required noninvasive mechanical ventilation. She then achieved a complete remission and received two additional courses of consolidation therapy. Her respiratory status remained stable and she is still alive with no evidence of leukemia two years after diagnosis.

\section{Patient No. 2}

A 46-year-old man complained of a sore throat and fever. He was diagnosed with pharyngitis and treated with levofloxacin and azithromycin. However, his symptoms worsened. Plain film chest X-ray revealed bilateral pulmonary infiltration. Blood tests showed an increased white blood cell count $(122,030 \mu \mathrm{L})$ with $84 \%$ myeloblasts. He was then referred to us and diagnosed with acute monocytic leukemia. The chemotherapy consisted of idarubicin, cytarabine and methylprednisolone (idarubicin, $12 \mathrm{mg} / \mathrm{m}^{2}$, administered on Days 7-9; cytarabine, $100 \mathrm{mg} / \mathrm{m}^{2}$, administered on Days 1-9; and methylprednisolone, $80 \mathrm{mg} /$ body on Days 1-9). He developed respiratory distress just after chemotherapy initiation and required oxygen support (up to 15 L/min) on Day 5 (Fig. 1-2). Chemotherapy was continued, and his respiratory status improved. Although the increase in the number of monoblasts was controlled, the patient did not enter a complete remission. However, after cord blood stem cell transplantation with myeloablative conditioning, he achieved a complete remission and did not experience any further episodes of respiratory failure. He is alive and well two years after the first admission.

\section{Patient No. 3}

A 66-year-old man complained of a two-week history of general fatigue and dyspnea. Blood tests showed a white blood cell count of $106,850 \mu \mathrm{L}$ with $64 \%$ myeloblasts. He was referred to our hospital and diagnosed with acute myelomonocytic leukemia with inv(16)(p13.1q22). The patient was scheduled to receive idarubicin and cytarabine therapy; however, his respiratory status deteriorated four hours after cytarabine infusion and his total bilirubin level began to increase. Therefore, idarubicin administration was cancelled and he was treated with cytarabine with noninvasive mechanical ventilation support (Fig. 1-3). His respiratory status progressively worsened and he was intubated on Day 6. Bronchoscopy was performed on the same day. 


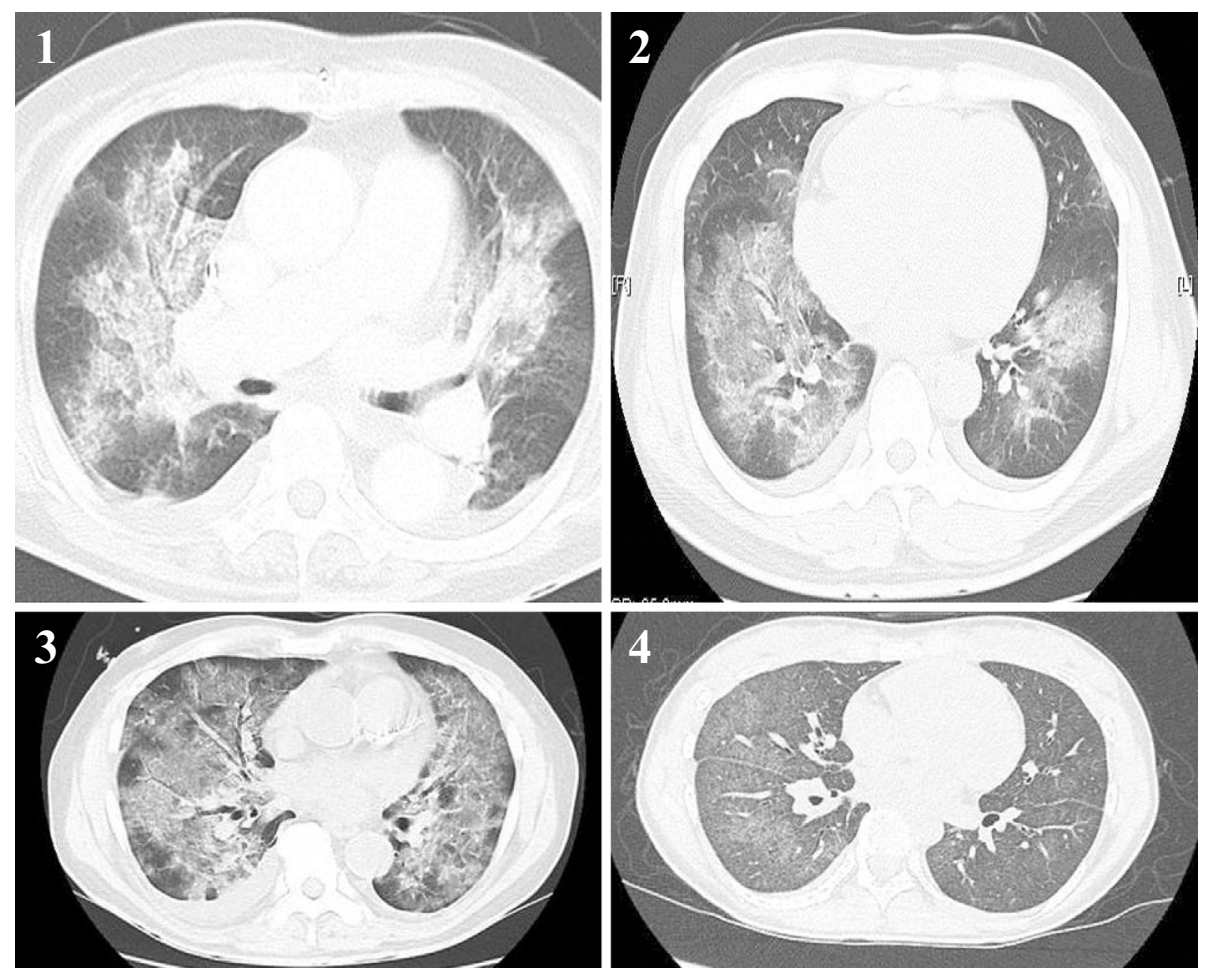

Figure 1. Figure 1-1. Patient 1: A CT scan performed on the first day of MEC therapy shows bilateral patchy areas of ground-glass opacity (GGO). Figure 1-2. Patient 2: A CT scan performed on the third day of idarubicin and cytarabine therapy shows bilateral areas of GGO. Figure 1-3. Patient 3: A CT scan performed on Day 4 of induction therapy shows bilateral ground-glass opacity/ infiltration. Figure 1-4. Patient 4: A chest CT scan obtained on the first day of induction chemotherapy shows right-sided lung dominant GGO.

Bronchoalveolar lavage (BAL) fluid obtained from the patient was not bloody, although it was different from that of intra-alveolar hemorrhage. BAL fluid (BALF) cultures for common bacteria, fungi and mycobacteria were negative. Multiplex polymerase chain reaction (PCR) was then performed to detect a fixed set of viruses, including Herpes simplex virus, Varicella zoster virus, Epstein-Barr virus, Cytomegalo virus (CMV) and Human herpesviruses (HHV)-6, HHV-7 and HHV-8. The results were negative.

However, the BALF contained numerous monoblasts (which were not present in the peripheral blood; Fig. 2). Etoposide $\left(100 \mathrm{mg} / \mathrm{m}^{2}\right)$ and methylprednisolone $(120 \mathrm{mg} /$ body) were added to the treatment regimen on Day 8 to restore the lung function. After the infusion of etoposide, the patient's respiratory status improved, and he was successfully extubated on Day 12 after chemotherapy initiation. He achieved a complete remission and received two courses of high-dose cytarabine as consolidation therapy. His respiratory status did not deteriorate again.

\section{Patient No. 4}

A 15-year-old girl was referred to our hospital after a week of fever that was refractory to antibiotics. Her white blood cell count was $7,700 \mu \mathrm{L}$ with $59 \%$ blasts. She was diagnosed with acute myeloid leukemia with $\operatorname{inv}(16)(\mathrm{p} 13.1 \mathrm{q} 22)$. Chemotherapy was initiated according to the AML99 protocol (etoposide, $150 \mathrm{mg} / \mathrm{m}^{2}$, administered on Days 1-5; cytarabine, $200 \mathrm{mg} / \mathrm{m}^{2}$, administered on Days 612; and mitoxantrone, $5 \mathrm{mg} / \mathrm{m}^{2}$, administered on Days 6-10, with an intrathecal injection on Day 6) (9). Shortly after etoposide initiation, she developed acute respiratory distress. Chest radiography showed progressive lung infiltration (Fig. 1-4). We continued chemotherapy and added methylprednisolone $(2 \mathrm{mg} / \mathrm{kg})$ to the regimen. The lung infiltrate improved seven days later. On Day 8, the patient received mitoxantrone and cytarabine, and her respiratory function remained stable. She achieved a complete remission and completed AML99 consolidation therapy.

\section{Discussion}

Pulmonary complications occurring within the early days after the initiation of chemotherapy in AML patients may result from various etiologic factors, including infection, intra-alveolar hemorrhage, pulmonary overload and chemotherapy toxicity. In all four of the present patients, no CMV antigens or DNA were detectable and the serum $\beta$-D glucan level was not increased, indicating that CMV and pneumocystis jiroveci pneumonia were unlikely causes of the ground grass opacity observed in these patients. In addition, their symptoms improved without the administration of therapy specific for these pathogens. Blood cultures for bacteria 


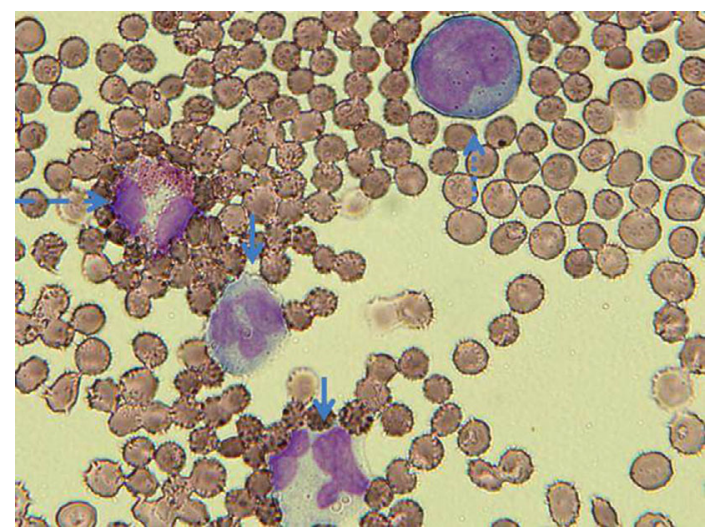

Figure 2. Patient 3: A cytospin preparation of bronchoalveolar lavage fluid (BALF) obtained on Day 6 after chemotherapy initiation. The solid arrows indicate mature monocytes, the dotted arrow indicates a monoblast and the dashed arrow indicates an eosinophil. 920 of the 1,000 cells in the BALF were positive for inv (16) according to a fluorescence in situ hybridization (FISH) analysis.

and fungi were also negative and sputum cultures showed only normal flora. Moreover, none of the four patients exhibited hemosputum or hemoptysis. Blood tests and echocardiography revealed that all patients had normal cardiac and renal functions. Although cytarabine often produces a unique clinicopathologic picture of noncardiogenic pulmonary edema, all patients received cytarabine as consolidation therapy and did not subsequently develop respiratory failure.

There are three known unusual pulmonary complications that are unique to leukemia: leukostasis, leukemic cell lysis pneumonopathy and hyperleukocytic reactions (2). Acute respiratory failure caused by increased leukocyte or blast cell counts is relatively common. Mckee et al. identified pulmonary vascular "leukostasis" or small vessel infiltration and occlusion by leukemic cell aggregates in all patients with a leukocyte count greater than $200,000 \mu \mathrm{L}$ (10). Myers et al. described a second type of reaction, termed "leukemic cell lysis pneumonopathy" (5), which causes local tissue damage and hypoxemia as a result of vascular obstruction and oxygen consumption by blast cells, with the injury being perpetuated by toxic and thromboplastic substances released by these cells in response to chemotherapy. Recently, it has been reported that LCLP can occur in patients with leukocyte counts of less than $50,000 / \mu \mathrm{L}(4,7,8)$. A third, similar condition (although the underlying mechanism is different) was reported by Vernant and co-workers (11). Pulmonary leukostasis, caused by the accumulation of blast cells in arterioles and capillaries, induces microhemorrhages and alveolar edema. Vernant et al. reported that the induction of a "hyperleukocytic" state is correlated with acute respiratory distress.

In the present patients, several mechanisms complicatedly interacted. The situation may not be the same among patients who do or do not exhibit hyperleukocytosis. In addition, it is difficult to distinguish pulmonary leukostasis and infiltration without evaluating tissue specimens. However, the fact that, in each case, respiratory distress developed shortly (within 18 hours) after chemotherapy initiation and continued chemotherapy administered to reduce the number of leukemic cells also successfully improved the pulmonary function, strongly favors LCLP as the primary cause of the pulmonary complications. Controversy exists regarding the use of steroids to treat LCLP. Steroids decrease the release of cytokines and oxidants, reduce blast adhesion to endothelial cells and prevent blast cell degeneration within the interstitium. Indeed, steroids have been used to prevent acute respiratory distress syndrome, albeit with variable results (8).

Bug et al. reported that AML patients with hyperleukocytosis who were treated with leukapheresis have a significantly reduced risk of early death (by Day 21 after chemotherapy initiation) compared with patients not treated with leukapheresis (12). Moreover, Ameri et al. suggested that leukapheresis may reduce the incidence of pulmonary hemorrhage in patients with severe leukocytosis (13). With hindsight, we should have considered the use of leukapheresis in Patient's No. 2 and 3; however, we doubt whether leukapheresis would have removed the leukemic cells that had already infiltrated the lungs.

Further studies including a greater number of patients with AML complicated by respiratory failure are needed.

The authors state that they have no Conflict of Interest (COI).

\section{Acknowledgement}

I would like to express my deepest gratitude to the anonymous reviewers who provided helpful comments and suggestions.

\section{References}

1. Chaoui D, Legrand $\mathrm{O}$, Roche $\mathrm{N}$, et al. Incidence and prognostic value of respiratory events in acute leukemia. Leukemia 18: 670$675,2004$.

2. Hildebrand FL, Rosenow EC, Habermann TM, Tazelaar HD. Pulmonary complications of leukemia. Chest 98: 1233-1239, 1990.

3. Kovalski R, Hansen-Flaschen J, Lodato RF, Pietra GG. Localized leukemic pulmonary infiltrates. Diagnosis by bronchoscopy and resolution with therapy. Chest 97: 674-678, 1990.

4. Tryka AF, Godleski JJ, Fanta CH. Leukemic cell lysis pneumopathy. A complication of treated myeloblastic leukemia. Cancer 50: 2763-2770, 1982.

5. Myers TJ, Cole SR, Klatsky AU, Hild DH. Respiratory failure due to pulmonary leukostasis following chemotherapy of acute nonlymphocytic leukemia. Cancer 51: 1808-1813, 1983.

6. Koh TT, Colby TV, Muller NL. Myeloid leukemias and lung involvement. Seminars in Respiratory Critical Care Medicine 26: 514-519, 2005.

7. Azoulay E, Fieux F, Moreau D, et al. Acute monocytic leukemia presenting as acute respiratory failure. Am J Respir Crit Care Med 167: 1329-1333, 2003.

8. Azoulay E, Canet E, Raffoux E, et al. Dexamethasone in patients with acute lung injury from acute monocytic leukaemia. Eur Respir J 39: 648-653, 2012.

9. Tsukimoto I, Tawa A, Horibe K, et al. Risk-stratified therapy and 
the intensive use of cytarabine improves the outcome in childhood acute myeloid leukemia: the AML99 trial from the Japanese Childhood AML Cooperative Study Group. J Clin Oncol 27: 4007-4013, 2009.

10. Mckee LC, Collins RD. Intravascular leukocyte thrombi and aggregates as a cause of morbidity and mortality in leukemia. Medicine 53: 463-478, 1974.

11. Vernant JP, Brun B, Mannoni P, Dreyfus B. Respiratory distress of hyperleukocytic granulocytc leukemias. Cancer 44: 264-268, 1979.

12. Bug G, Anargyrou $K$, Tonn $T$, et al. Impact of leukapheresis on early death rate in adult acute myeloid presenting with hyperleukocytosis. Transfusion 47: 1843-1850, 2007.

13. Al Ameri A, Koller C, Kantarjian H, et al. Acute pulmonary failure during remission induction chemotherapy in adults with acute myeloid leukemia or high-risk myelodysplastic syndrome. Cancer 116: $93-97,2010$.

(C) 2013 The Japanese Society of Internal Medicine http://www.naika.or.jp/imonline/index.html 